\title{
Preventing Radiobleaching of Cyanine Fluorophores Enhances Stability of Nuclear/NIRF Multimodality Imaging Agents
}

\author{
Reinier Hernandez ${ }^{1,2}$, Sandra Heskamp ${ }^{1}$, Mark Rijpkema ${ }^{1}$, Desirée L. Bos ${ }^{1}$, David M. Goldenberg 3 , William \\ J. McBride ${ }^{3}$, Alfred Morgenstern'4, Frank Bruchertseifer ${ }^{4}$, Weibo Cai ${ }^{2,5,6}$, Otto C. Boerman ${ }^{1 凶}$ \\ 1. Department of Radiology and Nuclear Medicine, Radboud University Medical Center, Nijmegen, The Netherlands. \\ 2. Department of Medical Physics, University of Wisconsin, Madison, Wisconsin, USA \\ 3. Immunomedics, Morris Plains, NJ, United States. \\ 4. European Commission, Joint Research Centre - Directorate for Nuclear Safety and Security, Karlsruhe, Germany. \\ 5. Department of Radiology, University of Wisconsin, Madison, WI, USA. \\ 6. University of Wisconsin Carbone Cancer Center, Madison, WI, USA. \\ $\triangle$ Corresponding author: email: Otto.Boerman@radboudumc.nl.
}

(C) Ivyspring International Publisher. Reproduction is permitted for personal, noncommercial use, provided that the article is in whole, unmodified, and properly cited. See http://ivyspring.com/terms for terms and conditions.

Received: 2016.01.28; Accepted: 2016.08.04; Published: 2017.01.01

\begin{abstract}
Despite the large interest in nuclear/optical multimodality imaging, the effect of radiation on the fluorescence of fluorophores remains largely unexplored. Herein, we report on the radiobleaching of cyanine fluorophores and describe conditions to provide robust radioprotection under practical (pre)clinical settings. We determined the radiosensitivity of several cyanine fluorescent compounds, including IRDye $800 \mathrm{CW}(800 \mathrm{CW})$ and a dual modality imaging tetrapeptide containing DOTA as chelator and Dylight 800 as fluorophore, exposed to increasing activities of ${ }^{111} \mathrm{In},{ }^{68} \mathrm{Ga}$, or ${ }^{213} \mathrm{Bi}(\mathrm{V}, \mathrm{EC} / \beta$, and $\alpha$ emitter, respectively). An activity and type of radiation-dependent radiation-induced loss of fluorescence, radiobleaching, of $800 \mathrm{CW}$ was observed upon incubation with escalating activities of ${ }^{111} \mathrm{In},{ }^{68} \mathrm{Ga}$, or ${ }^{213}{ }^{3}{ }^{6} .{ }^{68} \mathrm{Ga}$ showed the largest radiolytic effect, followed by ${ }^{111} \mathrm{In}$ and ${ }^{213} \mathrm{Bi}$. The addition of oxygen radical scavengers including ethanol, gentisic acid, and ascorbic acid (AA), provided a concentration dependent radioprotective effect. These results supported the hypothesis of a free radical-mediated radiobleaching mechanism. AA provided the most robust radioprotection over a wide range of concentrations and preserved fluorescence at much higher radioactivity levels. Overall, both near-infrared fluorescent compounds displayed similar sensitivity, except for ${ }^{213} \mathrm{Bi}$-irradiated solutions, where the dual modality construct exhibited enhanced radiolysis, presumably due to direct radiation damage from $\alpha$ particles. Concurrently, AA was not able to preserve fluorescence of the dual-modality molecule labeled with ${ }^{213} \mathrm{Bi}$. Our findings have important consequences for several research areas including ROS sensing, radiation-mediated drug release (uncaging), fluorescent dosimetry, and in the preparation of dual-modality radiopharmaceuticals.
\end{abstract}

Key words: radiobleaching, multimodality imaging, nuclear-optical imaging, radioprotection, radical scavengers.

\section{Introduction}

Multimodality molecular imaging agents bring together the strength of different imaging modalities to create multiplex probes that overcome the limitations of each single modality [1]. Perhaps, the best marriage is between optical imaging and nuclear imaging modalities such as positron emission tomography (PET) or single-photon emission tomography (SPECT). Such an approach combines the 
great temporal and spatial resolution provided by optical imaging with the exquisite tissue penetrability and excellent quantitation capabilities of nuclear techniques [2]. In general, nuclear/optical imaging agents feature a single or multiple fluorescent moieties -typically an organic dye- and chelators that can coordinate radiometals. Although the clinical application of such multimodality imaging approaches is still in its infancy, the intense research on the development of novel probes and detection systems is paving the way for the widespread clinical implementation of these technologies [2].

In the probe development front, we have extensive experience in the synthesis and implementation of nuclear/optical imaging agents, in both preclinical and clinical settings [3-5]. Despite the obvious advantages inherent to the multimodality paradigm, preparation and stability of multimodality agents may be challenging. Specifically, the fluorescent signal of the probes may be severely compromised when radiolabeled probes are stored, even for relatively short periods. Hence, understanding the effects of radiation on fluorophores is of paramount importance to avoid loss of fluorescence signal, which will certainly limit the applicability of the nuclear/optical approach. Here, we describe the effect of radiation exposure on the photostability of a series of near-infrared fluorescent compounds.

Given their advantageous properties as labels of biological molecules, carbocyanine dyes (e.g. Cy3, Cy5.5, Cy7, Dylight 800, and IRDye $800 \mathrm{CW}$ ) are among the most commonly used fluorophores employed in fluorescence-based biomedical assays [6]. From those, IRDye $800 \mathrm{CW}(800 \mathrm{CW})$ and its derivatives are a frequent choice for in vivo imaging, due to their long $\sim 800 \mathrm{~nm}$ emission wavelength which minimizes autofluorescence and enhances tissue penetrability $[7,8]$. Herein, we employed the native $800 \mathrm{CW}$ and a dual modality imaging tetrapeptide conjugated with DOTA and Dylight 800 (RDC018) (Fig. 1A), as a model to study the effects of radiation on the fluorescence of this type of organic molecules. Three radionuclides, ${ }^{68} \mathrm{Ga},{ }^{111} \mathrm{In}$, and ${ }^{213} \mathrm{Bi}$, which present significantly different properties (Fig. 1B) regarding decay mode, half-life, and emission energy were chosen as radiation sources.
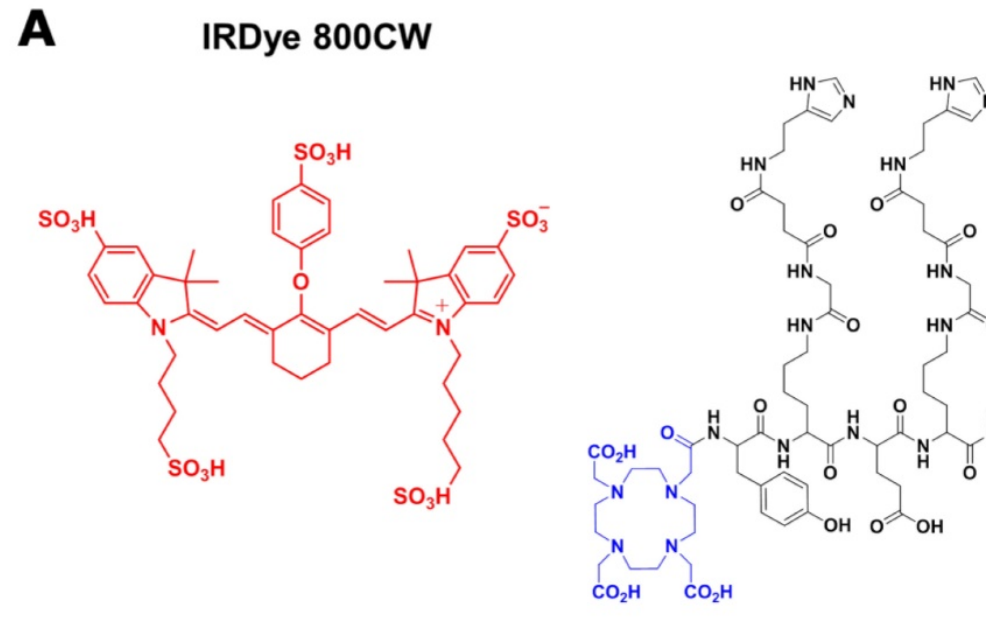

\section{RDC018}

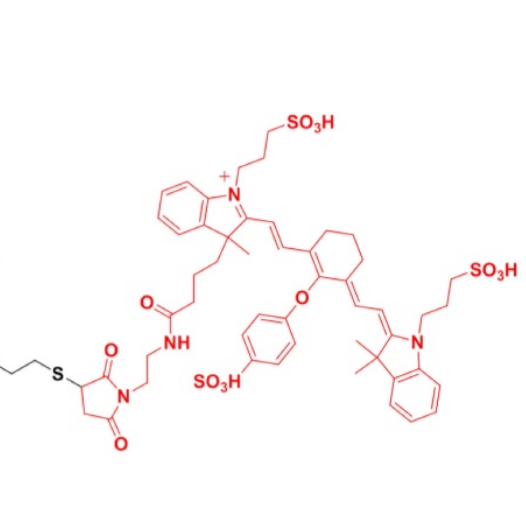

B
Radionuclide

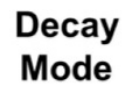
Half-life
$\gamma$ emissions (keV)
Dose to $\mathrm{H}_{2} \mathrm{O}$
$(\mathrm{mGy} / \mathrm{MBq})^{*}$
LET
$(\mathrm{keV} / \mu \mathrm{m})$

\begin{tabular}{cccccc}
\hline${ }^{111} \mathrm{In}$ & EC & $67.3(\mathrm{~h})$ & $\begin{array}{c}171.3(90.7 \%) \\
245.4(94.1 \%)\end{array}$ & 2230 & $0.2-0.3$ \\
& & & & \\
${ }^{68} \mathrm{Ga}$ & $\mathrm{EC} / \beta^{+}$ & $67.7(\mathrm{~min})$ & $511(177 \%)$ & 297 & $0.2-0.3$ \\
${ }^{213} \mathrm{Bi}$ & $\beta / / \alpha$ & $45.6(\mathrm{~min})$ & $440(25.9 \%)$ & 5280 & 140 \\
\hline
\end{tabular}

* Calculated integral dose to $1 \mathrm{cc}$ water sphere

Figure 1. Near-infrared fluorescent compounds and radionuclides employed in the study. A) Scheme showing the chemical structure of IRDye800CW and RDC018; the latter composed of Dylight 800 (red), a DOTA moiety for radiolabeling (blue) and an HSG targeting moiety (black). B) Relevant nuclear properties of IIIIn, 68 Ga, and ${ }^{213} \mathrm{Bi}$. The contribution of $213 \mathrm{Po}$ is considered in the ${ }^{213} \mathrm{Bi}$ dose. 
The optimal excitation/emission wavelengths of 800CW and RDC018 were determined by analyzing their spectra (Fig. S1A). For both compounds, $740 \mathrm{~nm}$ and $796 \mathrm{~nm}$ were selected as excitation and emission wavelength, respectively. The influence of several experimental conditions including buffer, $\mathrm{pH}$, heating, and the addition of metals on $800 \mathrm{CW}^{\prime} \mathrm{s}$ fluorescence emission at $796 \mathrm{~nm}$ was determined (Fig. S1B-D). A marked difference in fluorescent intensity was noted when $800 \mathrm{CW}$ was buffered with NaAc, MES, or HEPES. NaAc buffer was employed in all subsequent studies given that $800 \mathrm{CW}$ fluorescence was strongest under such conditions. Lowering the $\mathrm{pH}$ of the solution resulted in a loss of fluorescence intensity due to the protonation of the indolic nitrogen, and prolonged heating of $800 \mathrm{CW}$ at $95{ }^{\circ} \mathrm{C}$ provoked a linear decrease in fluorescence intensity; fluorescent intensities were down to $60 \%$ of the initial values after one $h$ at $95{ }^{\circ} \mathrm{C}$. Finally, the effect of non-radioactive metals on $800 \mathrm{CW}$ fluorescence was tested (Fig. S2). No significant difference $(P>0.05)$ in fluorescence signal was observed after incubation of $800 \mathrm{CW}$ with $\mathrm{Ga}^{3+}(6.6 \mathrm{pg})$ and $\mathrm{In}^{3+}(1.3 \mathrm{ng})$; these concentrations resembled those of the corresponding radioactive metals.

Aqueous solutions of $800 \mathrm{CW}$ in $0.1 \mathrm{M} \mathrm{NaAc}(\mathrm{pH}$ $4.5)$, were exposed to increasing activities of ${ }^{68} \mathrm{Ga}$, ${ }^{111} \mathrm{In}$, and ${ }^{213} \mathrm{Bi}$. The effect of each type of radiation at different activity concentrations was assessed by measuring the fluorescence of the dye - normalized to a non-irradiated control - at different time points. A pronounced dose-dependent decrease in $800 \mathrm{CW}$ fluorescence was observed after $30 \mathrm{~min}$ exposure to ${ }^{68} \mathrm{Ga}$ (Fig. 2A). ${ }^{111} \mathrm{In}$ and ${ }^{213} \mathrm{Bi}$ presented milder effects at early time points, but prolonged exposures to ${ }^{111} \mathrm{In}$ (e.g. $24 \mathrm{~h}$ ) also resulted in a significant deterioration of the fluorescence signal (Fig. 2B and Fig. S3). Matrix-assisted desorption ionization time-of-flight (MALDI-TOF) mass spectrometry showed the disappearance of the mass peak corresponding to the intact $800 \mathrm{CW}(\mathrm{m} / \mathrm{z}=1003.2)$, in the irradiated vs. the non-irradiated samples (Fig. 2C and Fig. 2D), demonstrating the non-reversible radiation-induced degradation of the fluorophore. Unfortunately, due to the complexity of the resulting mass spectrum in the irradiated samples, fragmentation analysis was unfeasible.

At similar activity level and exposure time, the extent of radiobleaching for the three radionuclides followed the trend ${ }^{68} \mathrm{Ga}>{ }^{111} \mathrm{In}>{ }^{213} \mathrm{Bi}$. Strong ${ }^{68} \mathrm{Ga}$-induced radiobleaching was also evident in near-infrared fluorescent (NIRF) images, where a notably lower signal was recorded for ${ }^{68} \mathrm{Ga}$-irradiated solutions (Fig. S4A and B). Based on our dose estimations, ${ }^{213} \mathrm{Bi}$ should have elicited radiobleaching effects that surpassed those observed for ${ }^{68} \mathrm{Ga}$ (Table S1). Such detachment between the estimated dose imparted by the radionuclide and the extent of radiobleaching suggests that the loss of fluorescence was a secondary effect, not mediated by the direct interaction of the fluorophore with the ionizing radiation.

The mechanism of water radiolysis leading to the formation of highly reactive $\mathrm{H}^{\cdot}, \mathrm{OH}$, and $\mathrm{e}^{-}$aq radicals is now well understood. Amongst all generated reactive oxygen species (ROS), hydroxyl radicals are the primary oxidizing entities produced during water radiolysis. Such reactive species readily participate in addition reactions $\left(\mathrm{k} \sim 10^{10} \mathrm{M}^{-1} \mathrm{~S}^{-1}\right)$ in the presence of conjugated or aromatic organic molecules and are responsible for the observed degradation of organic chromophores after irradiation in aqueous solution [9, 10]. Similarly, scientists have recently described the mechanism of degradation of heptamethine cyanine dyes via oxidative processes involving primarily singlet oxygen $\left({ }^{1} \mathrm{O}_{2}\right)$ radicals [11-13]. These studies demonstrated that the sensitivity of cyanine dyes is dictated by both the radical reactivity and the oxidation potential of the dye. The latter is inextricably ligated to the length of the polymethine chain and the presence of electron withdrawing substituents in the indolenine moiety [14-16].

A $\mathrm{OH}$ radical mediated radiobleaching mechanism explains the low radiolytic effects observed after ${ }^{213} \mathrm{Bi}$ irradiation of $800 \mathrm{CW}$ solutions. During water radiolysis, hydroxyl radical yields, $\mathrm{G}\left({ }^{\circ} \mathrm{OH}\right)$, are highly dependent on the linear energy transfer (LET) of the incident radiation. High LET radiation (e.g. a and heavy ions) generate a densely ionized media that promotes the recombination of $\mathrm{OH}$ radical to form less reactive molecular species (e.g. $\mathrm{H}_{2} \mathrm{O}_{2}$ ), which results in significantly lower $\mathrm{G}\left({ }^{\circ} \mathrm{OH}\right)[17,18]$. Despite ${ }^{213} \mathrm{Bi}$ decays by $\beta$, it is in secular equilibrium with its radioactive daughter, ${ }_{213} \mathrm{Po}$, a pure a emitter $\left(\mathrm{t}_{1 / 2}: 3.7 \mu \mathrm{s}, \mathrm{E}_{\alpha}=8.376 \mathrm{MeV}\right)$. The a radiation from ${ }^{213} \mathrm{Po}$ delivers more than $98 \%$ of the dose imparted by the isotopic pair. However, due to its high LET, G('OH) associated with ${ }^{213}$ Po radiation are significantly lower, minimizing any $\cdot \mathrm{OH}$-promoted radiobleaching processes.

To corroborate the hypothesis of a $\mathrm{OH}$ radical mediated degradation of the fluorophores, we determined the effect of the addition of radical scavengers - a strategy that has been widely employed for the protection of organic molecules against free radical-mediated damage - on the radiobleaching of $800 \mathrm{CW}$ solutions [19-21]. Normalized $800 \mathrm{CW}$ fluorescence was determined after $0.5,3$ and $6 \mathrm{~h}$ exposure to $20 \mathrm{MBq}$ of ${ }^{68} \mathrm{Ga}$ in the presence of increasing concentrations of gentisic acid 
(GA), ethanol (EtOH), and ascorbic acid (AA) (Fig. 3A-C). All scavengers provided some degree of radioprotection, which in the case of GA and EtOH was both concentration and exposure time dependent. Only AA was able to preserve completely $800 \mathrm{CW}$ fluorescence after longer radiation exposure, in a much wider range of concentrations (0.001-10 \% $(\mathrm{w} / \mathrm{v}))$. NIRF images in Fig. S4 reiterate the strong radioprotective effect of relatively low AA concentrations, $0.1 \%(\mathrm{w} / \mathrm{v})$, which were able to preserve 800CW's fluorescent signal. AA also provided effective radioprotection against ${ }^{111} \mathrm{In}$ and ${ }^{213} \mathrm{Bi}$ induced radiobleaching (Fig. 3D and Fig. S5). The superior radioprotective properties of AA can be attributed to the very high rate constant $\left(\mathrm{k}=1.1 \times 10^{10}\right.$ L. mol $\left.^{-1} . \mathrm{s}^{-1}\right)$ observed in the reaction of $\mathrm{OH}$ radicals with AA acid, which is almost one order of magnitude higher than for GA and EtOH [22-24]. Additionally, AA scavenging properties are enhanced by its ability to stabilize two free radicals (Fig. 3E).

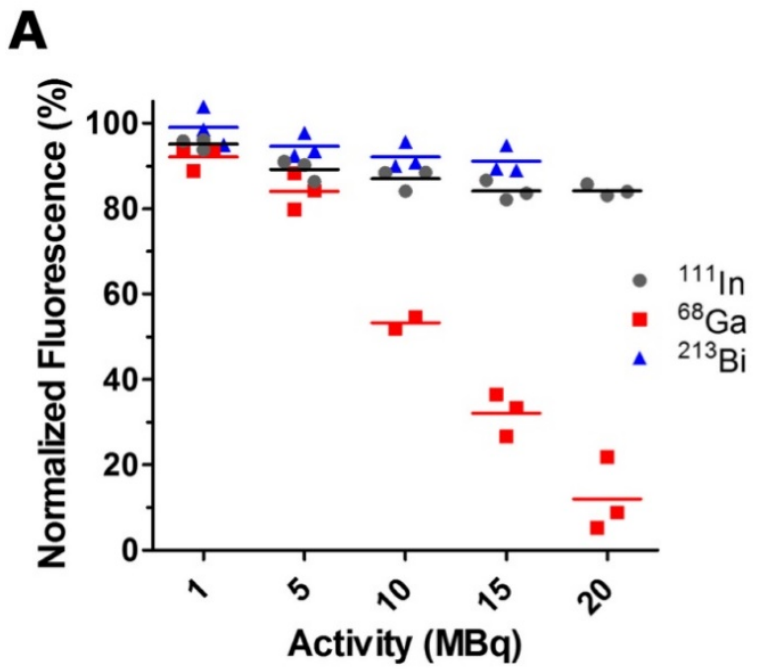

B
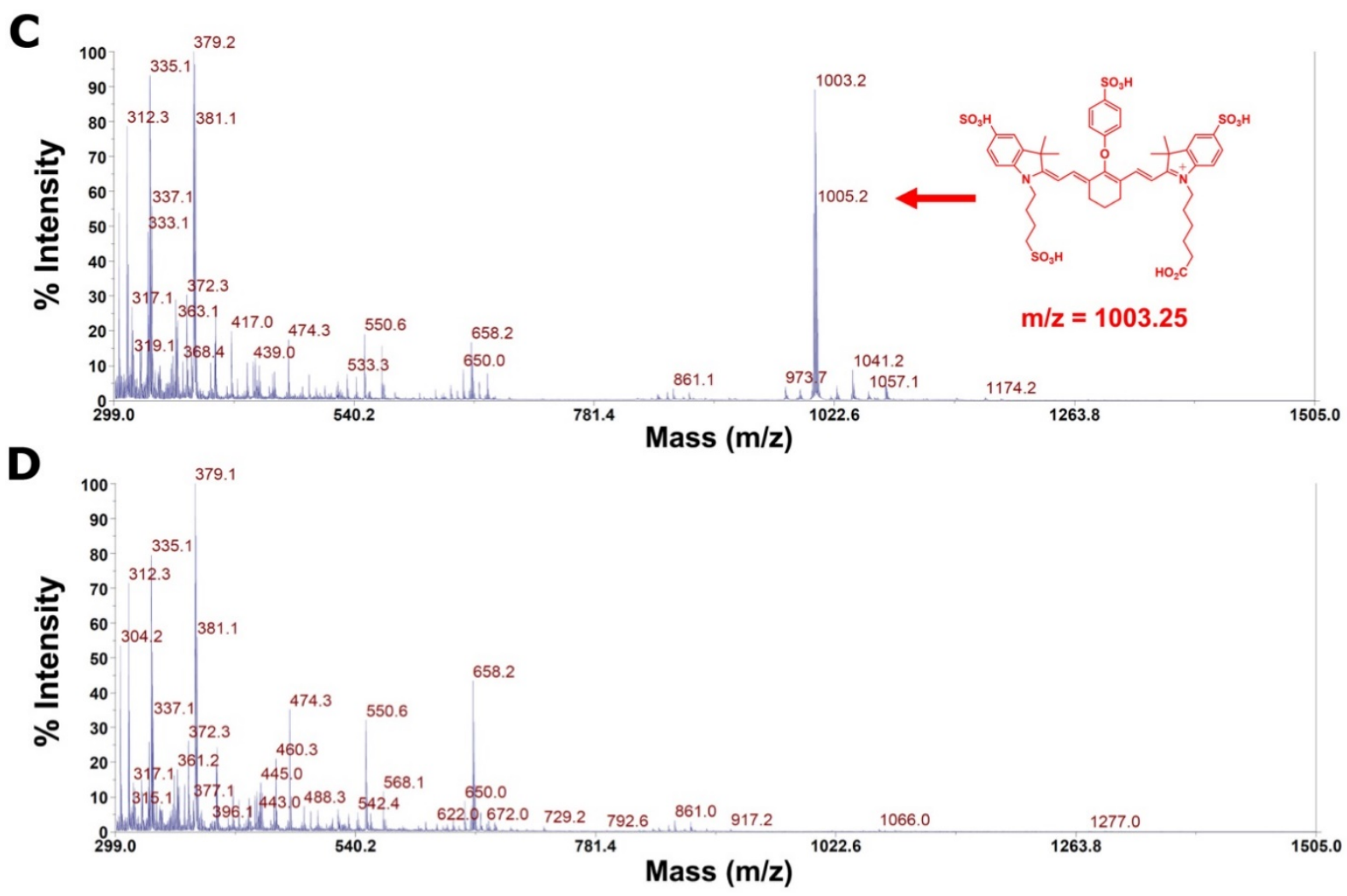

Figure 2. Photostability of irradiated IRDye $800 \mathrm{CW}$ solutions. A) Normalized $800 \mathrm{CW}$ fluorescence, 30 min after exposure to increasing activities of $111 \mathrm{In}$, $68 \mathrm{Ga}$, and $213 \mathrm{Bi}$. B) Effect of elapsed time in the radiobleaching of $800 \mathrm{CW}$ solutions irradiated with 1-20 MBq of $111 \mathrm{ln}$. MALDI-TOF mass spectrum of (C) $800 \mathrm{CW}$ and (D) $800 \mathrm{CW}$ after incubation with ${ }^{68} \mathrm{Ga}(20 \mathrm{MBq})$. The mass peak corresponding to $800 \mathrm{CW}(\mathrm{m} / \mathrm{z}=1003.2)$ clearly disappear after irradiation. 
A

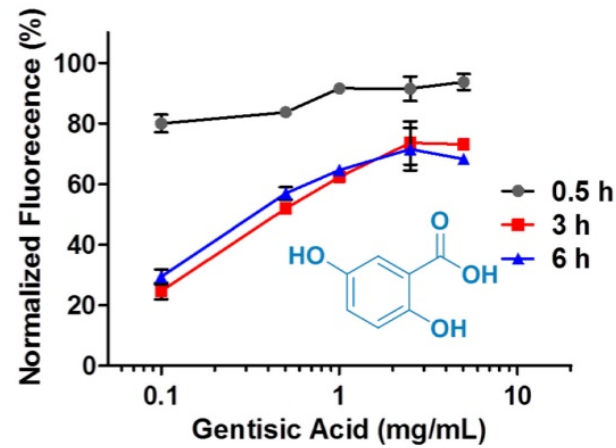

C

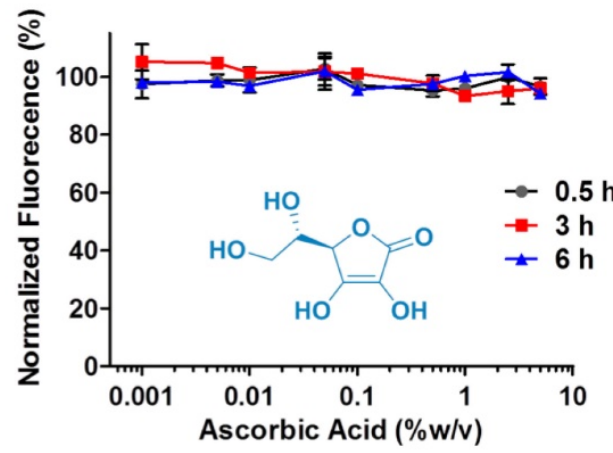

B

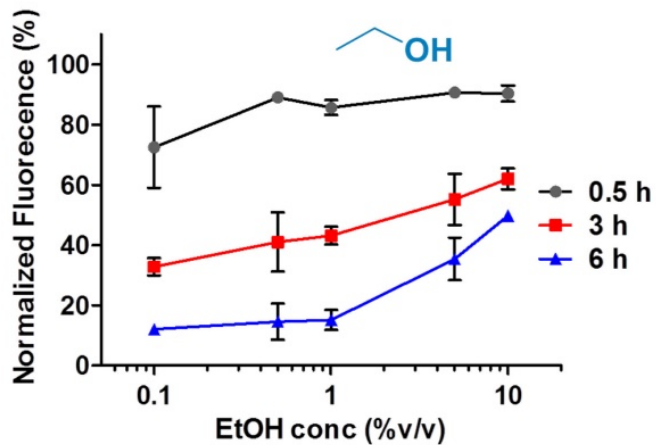

D

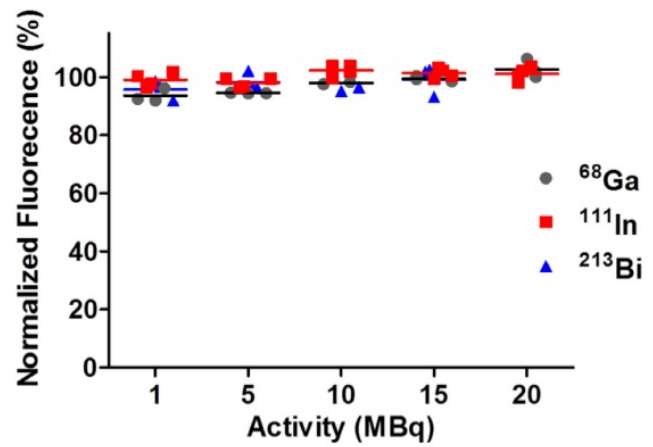

E<smiles>[X]CCOC1=C(O)C(=O)OC1[C@H](O)CO</smiles><smiles>CCOC(=O)C1OC(CO)C(=O)C1=O</smiles><smiles>O=C1OC(CO)C(=O)C1O</smiles><smiles>[X]CC1(O)C(=O)O[C@@H](C(O)CO)C1=O</smiles>

Figure 3. Radioprotective effects of ' $\mathrm{OH}$ radical scavengers against ${ }^{68 \mathrm{Ga}}$-promoted radiobleaching. Radioprotection curves for (A) GA, (B) EtOH, and (C) AA. D) AA $(0.1 \% \mathrm{w} / \mathrm{v})$ provides robust radioprotection against ${ }^{68} \mathrm{Ga},{ }^{11} 1 \mathrm{In}$, and ${ }^{21}{ }^{13} \mathrm{Bi}$ radiation. E) Scheme representing the mechanism of radical stabilization by AA.

To investigate whether other fluorophores are as sensitive to radiobleaching, the phenomenon was also investigated in three other common cyanine fluorescent dyes with different polymethine chain lengths (Fig. 4A). The results of a two-hour irradiation of Cy7, Cy5.5, and Cy3 with ${ }^{68} \mathrm{Ga}(10 \mathrm{MBq})$ revealed a marked difference in radiosensitivity between dyes (Fig. 4B). The order in radiosensitivity observed, Cy7, $800 \mathrm{CW}>\mathrm{Cy} 5.5>\mathrm{Cy} 3$, perfectly aligned with the results obtained by Nagano et al., which demonstrated an inverse correlation between the oxidation potential of the dye - a measure of radiosensitivity - and the length of the polymethine chain [14]. The radioprotective properties of AA were generalized for the entire series of dyes given that the addition of the radical scavenger invariably resulted in the inhibition of the radiation-induced fluorophore breakdown.

RDC018 is a nuclear/optical targeting agent that holds great promise for pretargeted cancer imaging and pretargeted radionuclide therapy [3]. RDC018 
features an NIR fluorescent moiety -with structural and spectral similarity to $800 \mathrm{CW}$ - and a chelating group for the conjugation of radiometals (Fig. 1A). Our next step was to investigate the impact of the direct attachment of the radionuclides on the fluorescence of the dual modality construct. This time, we used experimental conditions that resembled the actual preparation of radiolabeled RDC018 for preclinical studies. RDC018 was incubated at $95^{\circ} \mathrm{C}$ for 15 min with much higher activity levels: $350 \mathrm{MBq}(\sim 10$ $\mathrm{mCi})$ of ${ }^{68} \mathrm{Ga}, 116 \mathrm{MBq}(\sim 3 \mathrm{mCi})$ of ${ }^{111} \mathrm{In}$, and $30 \mathrm{MBq}$ ( $\sim \mathrm{mCi})$ of ${ }^{213} \mathrm{Bi}$, respectively. This experiment intended to mimic practical radiolabeling conditions, where radiobleaching effects are expected to be more drastic. As expected, after $6 \mathrm{~h}$ of incubation with either radionuclide, a complete loss of RDC018's fluorescence was observed (Fig. 4A). This corroborated the similarities between $800 \mathrm{CW}$ and RDC018 in terms of radiostability.

In another set of studies, an intermediate concentration of $0.1 \%(\mathrm{w} / \mathrm{v})$ of AA was added to the solutions before exposure to radiation. As observed with $800 \mathrm{CW}$, the fluorescence signal was quantitatively preserved in ${ }^{68} \mathrm{Ga}$ and ${ }^{111}$ In-irradiated samples. Interestingly, solutions exposed to ${ }^{213} \mathrm{Bi}$ still showed a $\sim 40 \%$ decline in fluorescence even in the presence of AA (Fig. 4B). These results indicated that ${ }^{213} \mathrm{Bi}$-induced radiobleaching of RDC018 was not solely determined by interaction with ${ }^{\circ} \mathrm{OH}$ radicals. Detailed experiments describing the radiobleaching of RDC018 with ${ }^{213} \mathrm{Bi}$ are shown in Fig. 4C. Although the exposure to ${ }^{213} \mathrm{Bi}$ did not result in a complete loss of fluorescence, at similar activity concentrations, RDC018 radiobleaching was more pronounced than the observed for $800 \mathrm{CW}$ (Fig. 4D). These findings, together with the fact that AA was only able to provide limited radioprotection suggest the direct interaction of a particles with RDC018 molecule. The chelation of ${ }^{213} \mathrm{Bi}$ by DOTA likely plays a vital role on the enhanced radiosensitivity RDC018, since the proximity of the fluorophore to the chelated ${ }^{213} \mathrm{Bi}$, increases the probability of a direct ionization of the fluorophore by a particles. Similar results have been widely reported in radiobiology, where radiation damage from high-LET a particles occurs via the direct ionization of the DNA molecule, independently of the presence of reactive radical species (oxygen enhancement ratio, OER 1) $[25,26]$.

To date, the relevance of radiobleaching within the context of molecular imaging remains overlooked. To the best of our knowledge, the radiostability of commonly used cyanine fluorescent dyes has not been systematically investigated before. Herein, we have shown that this family of fluorophores, particularly those that absorb light in the NIR range, are highly susceptible to radiobleaching, even at relatively low activity concentrations. Such phenomenon has been proven of immense potential for in vivo ROS sensing, fluorescence-based dosimetry, and, more recently, for the radical-facilitated release (uncaging) of cytotoxic agents from radio/photo-sensitive antibody-drug conjugates [14, 27]. Importantly, we also demonstrated that radioprotection is achievable through the addition of radical scavengers to the irradiated solution, even at the high activity levels required for preclinical/clinical imaging studies. Such a simple and versatile strategy will be of great impact for improving the application of nuclear/optical dual modality imaging agents; an aspect of vital importance for future successful clinical application where high stability of the imaging probes is required.
$\mathbf{A}$
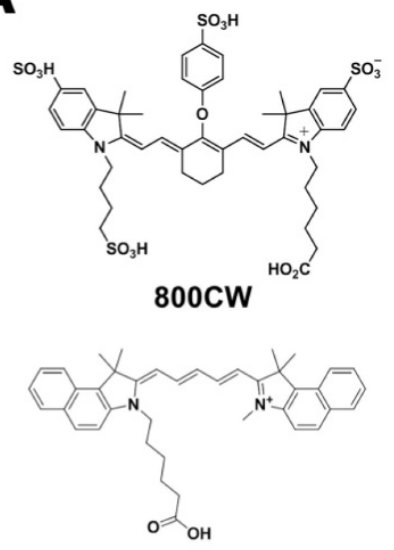

Cy5.5
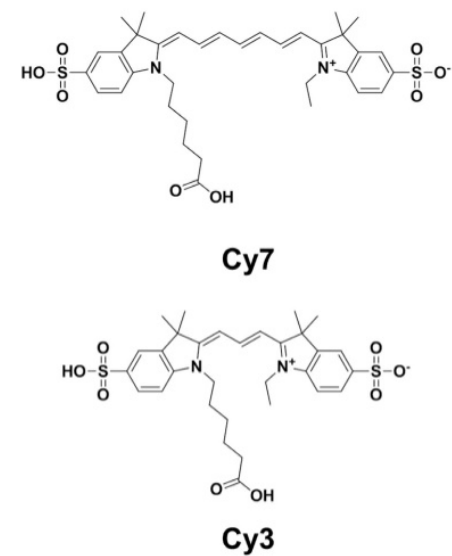

B

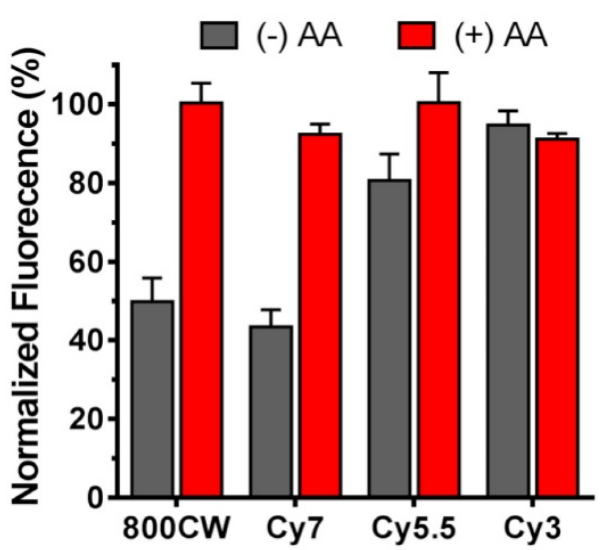

Figure 4. A) Structure of four common cyanine dyes: $800 \mathrm{CW}, \mathrm{Cy} 7, \mathrm{Cy} 5.5$, and Cy3. B) ${ }^{68 \mathrm{Ga}}$-induced radiobleaching of the dyes in the presence $(+)$ or absence $(-)$ of ascorbic acid $(0.1 \% \mathrm{w} / \mathrm{v})$. The dyes showed markedly different radiosensitivities: Cy7, $800 \mathrm{CW}>\mathrm{Cy} 5.5>\mathrm{Cy} 3$. 
A

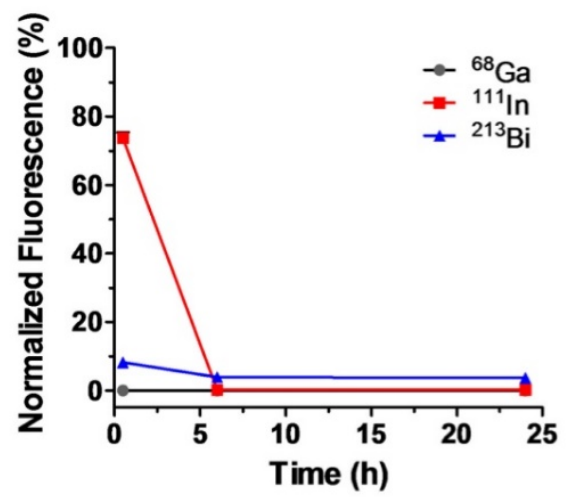

C

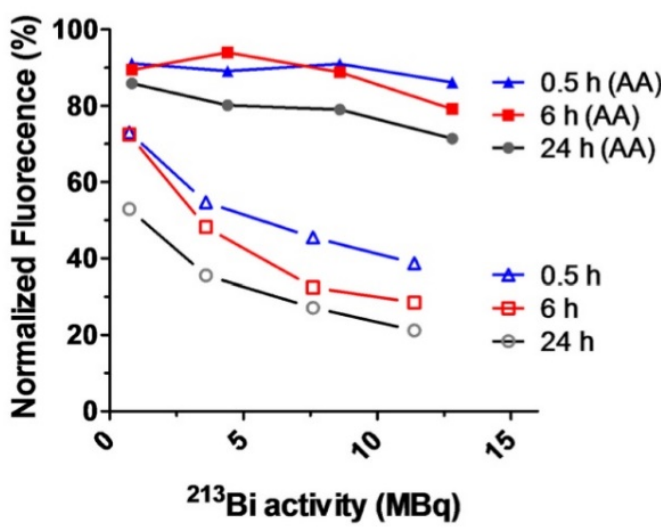

B

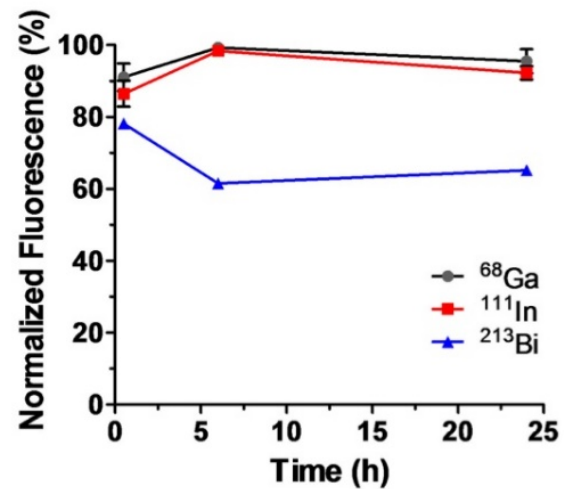

D

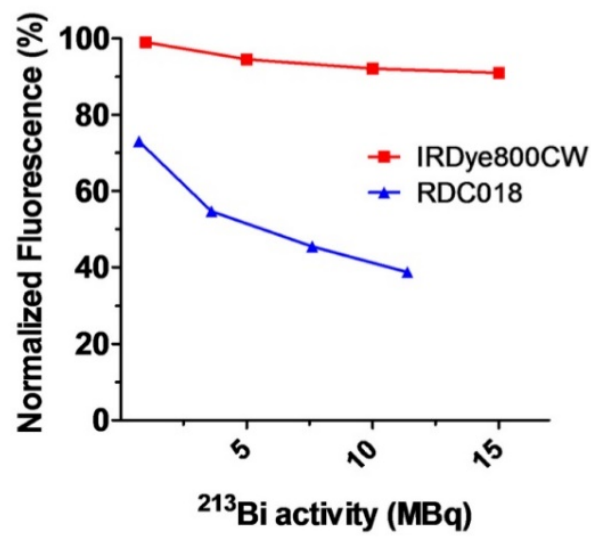

Figure 5. Photostability and radioprotection of RDC018. Radiobleaching (A) and $0.1 \%(w / v)$ AA radioprotection (B) of RDC018 exposed to elevated activities of ${ }^{68} \mathrm{Ga}$, $111 \mathrm{ln}$, and ${ }^{213} \mathrm{Bi}$. (C) Activity-dependent radiobleaching (open symbols) and $0.1 \%$ (w/v) AA radioprotection (filled symbols) of $213 \mathrm{Bi}$-labeled RDC018 solutions. Complete preservation of RDC018 fluorescence was not achieved in this case (D) Comparison of IRDye800CW vs. RDC018 radiobleaching upon $213 \mathrm{Bi}$ irradiation. RDC018 displayed significantly reduced radiostability due to direct interaction with $\alpha$ particles.

\section{Supplementary Material}

Supplementary figures and tables. http://www.thno.org/v07p0001s1.pdf

\section{Acknowledgment}

The authors thank Dr. Feng Chen for his insightful comments on the manuscript and Hector F. Valdovinos for his assistance with isotope production. Special thanks to the Graduate Research Opportunity Worldwide (GROW) program and the Netherlands Organisation for Scientific Research (NWO) for auspicing this research. This work was supported, in part, the National Science Foundation (DGE-1256259), and the National Institutes of Health (5T32GM08349).

\section{Competing Interests}

The authors have declared that no competing interest exists.

\section{References}

1. Jennings LE, Long NJ. 'Two is better than one'--probes for dual-modality molecular imaging. Chemical communications. 2009: 3511-24.
2. Culver J, Akers W, Achilefu S. Multimodality molecular imaging with combined optical and SPECT/PET modalities. Journal of nuclear medicine : official publication, Society of Nuclear Medicine. 2008; 49: 169-72.

3. Lutje S, Rijpkema M, Goldenberg DM, van Rij CM, Sharkey RM, McBride WJ, et al. Pretargeted dual-modality immuno-SPECT and near-infrared fluorescence imaging for image-guided surgery of prostate cancer. Cancer research. 2014; 74: 6216-23.

4. Rijpkema M, Oyen WJ, Bos D, Franssen GM, Goldenberg DM, Boerman OC. SPECT- and fluorescence image-guided surgery using a dual-labeled carcinoembryonic antigen-targeting antibody. Journal of nuclear medicine : official publication, Society of Nuclear Medicine. 2014; 55: 1519-24.

5. Lutje S, Rijpkema M, Franssen GM, Fracasso G, Helfrich W, Eek A, et al. Dual-Modality Image-Guided Surgery of Prostate Cancer with a Radiolabeled Fluorescent Anti-PSMA Monoclonal Antibody. Journal of nuclear medicine : official publication, Society of Nuclear Medicine. 2014; 55: 995-1001.

6. Gorka AP, Nani RR, Schnermann MJ. Cyanine polyene reactivity: scope and biomedical applications. Organic \& biomolecular chemistry. 2015; 13: 7584-98.

7. Azhdarinia A, Ghosh P, Ghosh S, Wilganowski N, Sevick-Muraca EM. Dual-labeling strategies for nuclear and fluorescence molecular imaging: a review and analysis. Molecular imaging and biology : MIB : the official publication of the Academy of Molecular Imaging. 2012; 14: 261-76.

8. Adams KE, Ke S, Kwon S, Liang F, Fan Z, Lu Y, et al. Comparison of visible and near-infrared wavelength-excitable fluorescent dyes for molecular imaging of cancer. Journal of biomedical optics. 2007; 12: 024017.

9. Dorfman, L., Adams, G. Reactivity of the Hydroxyl Radical in Aqueous Solutions. NSRDS-NBS. 1973; 46: 1-59.

10. Rauf MA, Ashraf SS. Radiation induced degradation of dyes--an overview. Journal of hazardous materials. 2009; 166: 6-16.

11. Stennett EM, Ciuba MA, Levitus M. Photophysical processes in single molecule organic fluorescent probes. Chemical Society reviews. 2014; 43: 1057-75.

12. Zheng Q, Jockusch S, Zhou Z, Blanchard SC. The contribution of reactive oxygen species to the photobleaching of organic fluorophores. Photochemistry and photobiology. 2014; 90: 448-54.

13. Nani RR, Kelley JA, Ivanic J, Schnermann MJ. Reactive Species Involved in the Regioselective Photooxidation of Heptamethine Cyanines. Chemical science. 2015; 6: 6556-63. 
14. Oushiki D, Kojima H, Terai $\mathrm{T}$, Arita $\mathrm{M}$, Hanaoka $\mathrm{K}$, Urano $\mathrm{Y}$, et al. Development and application of a near-infrared fluorescence probe for oxidative stress based on differential reactivity of linked cyanine dyes. Journal of the American Chemical Society. 2010; 132: 2795-801.

15. Chen X, Peng X, Cui A, Wang B, Wang L, Zhang R. Photostabilities of novel heptamethine $3 \mathrm{H}$-indolenine cyanine dyes with different $\mathrm{N}$-substituents. Journal of Photochemistry and Photobiology A: Chemistry. 2006; 181: 79-85.

16. Kundu K, Knight SF, Willett N, Lee S, Taylor WR, Murthy N. Hydrocyanines: a class of fluorescent sensors that can image reactive oxygen species in cell culture, tissue, and in vivo. Angewandte Chemie. 2009; 48: 299-303.

17. Laverne JA. The Production of Oh Radicals in the Radiolysis of Water with He-4 Ions. Radiat Res. 1989; 118: 201-10.

18. Burns WG, Sims HE. Effect of radiation type in water radiolysis. Journal of the Chemical Society, Faraday Transactions 1: Physical Chemistry in Condensed Phases. 1981; 77: 2803-13.

19. Joshi R, Gangabhagirathi R, Venu S, Adhikari S, Mukherjee T. Antioxidant activity and free radical scavenging reactions of gentisic acid: in-vitro and pulse radiolysis studies. Free radical research. 2012; 46: 11-20.

20. Liu S, Ellars CE, Edwards DS. Ascorbic acid: useful as a buffer agent and radiolytic stabilizer for metalloradiopharmaceuticals. Bioconjugate chemistry. 2003; 14: 1052-6.

21. Feierman DE, Winston GW, Cederbaum AI. Ethanol oxidation by hydroxyl radicals: role of iron chelates, superoxide, and hydrogen peroxide. Alcoholism, clinical and experimental research. 1985; 9: 95-102.

22. Buxton GV, Greenstock CL, Helman WP, Ross AB. Critical Review of rate constants for reactions of hydrated electrons, hydrogen atoms and hydroxyl radicals $(\cdot \mathrm{OH} / \cdot \mathrm{O}-$ in Aqueous Solution. Journal of Physical and Chemical Reference Data. 1988; 17: 513-886.

23. Oturan MA, Pinson J, Deprez D, Terlain B. Polyhydroxylation of salicylic acid by electrochemically generated $\mathrm{OH}$ radicals. New Journal of Chemistry. 1992; 16: 705-10.

24. Redpath JL,Willson R.L. Reducing compounds in radioprotection and radiosensitization: model experiments using ascorbic acid. Int J Radiat Biol Relat Stud Phys, Chem Med. 1973; 23: 51-65.

25. Barendsen GW. Parameters of linear-quadratic radiation dose-effect relationships: dependence on LET and mechanisms of reproductive cell death. International journal of radiation biology. 1997; 71: 649-55.

26. Wenzl T, Wilkens JJ. Modelling of the oxygen enhancement ratio for ion beam radiation therapy. Physics in medicine and biology. 2011; 56: 3251-68.

27. Nani RR, Gorka AP, Nagaya T, Kobayashi H, Schnermann MJ. Near-IR Light-Mediated Cleavage of Antibody-Drug Conjugates Using Cyanine Photocages. Angewandte Chemie. 2015; 54: 13635-8. 